Phys. Rev. D 94, 085025 (2016)

arXiv:1607.02099

\title{
Changes in extensive air showers from isotropic Lorentz violation in the photon sector
}

\author{
J.S. Díaz* and F.R. Klinkhament \\ Institute for Theoretical Physics, Karlsruhe Institute \\ of Technology (KIT), 76128 Karlsruhe, Germany \\ M. Risse‡ \\ Physics Department, University of Siegen, 57068 Siegen, Germany
}

\begin{abstract}
We consider a theory with isotropic nonbirefringent Lorentz violation in the photon sector and explore the effects on the development of the electromagnetic component of extensive air showers in the Earth atmosphere. Specifically, we consider the case of a "fast" photon with a phase velocity larger than the maximum attainable velocity of a massive Dirac fermion (this case corresponds to a negative Lorentz-violating parameter $\kappa$ in the action). Shower photons with above-threshold energies decay promptly into electron-positron pairs, instead of decaying by the conventional production of electron-positron pairs in the background fields of atomic nuclei. This rapid production of charged leptons accelerates the shower development, decreasing the atmospheric depth of the shower maximum $\left(X_{\max }\right)$ by an amount which could be measured by cosmic-ray observatories. Precise measurements of $X_{\max }$ could then improve existing limits on the negative Lorentz-violating parameter $\kappa$ by several orders of magnitude.
\end{abstract}

PACS numbers: 11.30.Cp, 12.20.-m, 13.85.Tp, 98.70.Sa

\footnotetext{
* jorge.diaz@kit.edu

$\dagger$ frans.klinkhamer@kit.edu

$\ddagger$ risse@hep.physik.uni-siegen.de
} 


\section{INTRODUCTION}

Ever since the foundation of special relativity, electrodynamics has played a fundamental role in the establishment of elementary-particle-physics theory. Experimental tests using photons have provided valuable and sensitive probes for the search of potential deviations from exact Lorentz symmetry. In fact, some of the most stringent limits on dimensionless parameters controlling Lorentz violation have been found in the photon sector. Although laboratory experiments have access to some unique signatures, the determination of most of the best limits on Lorentz-violating (LV) parameters in various sectors have taken advantage of the high energies or the large propagation distances of "astroparticles," i.e., cosmic rays [14], gamma rays [3], cosmic-microwave-background photons [5], and neutrinos [6, 7].

Here, we explore the potential of ultrahigh-energy photons to test LV effects and consider photons which are produced as secondary particles in air showers. In Sec. III, we give the theoretical setup for isotropic Lorentz violation in the photon sector and review existing bounds on the relevant LV parameter. In Sec. III, we discuss a simple model for the electromagnetic component of extensive air showers, first for the standard Lorentz-invariant theory and, then, for the Lorentz-violating theory considered. In Sec. IV, we present some concluding remarks.

As the scope of this article is restricted to the study of isotropic Lorentz violation in electrodynamics, we only consider isotropic Lorentz-violating effects in the photon sector. Notice that the corresponding effects from the electron sector can be moved to the photon sector by suitable spacetime coordinate transformations [8]. Furthermore, independent studies regarding Lorentz-violating effects involving weakly interacting particles can be found for muons [9], pions [10 12], and neutrinos [13].

\section{THEORY}

A relatively simple extension of standard quantum electrodynamics 14] adds a single LV term [15, 16] to the Lagrangian density. This term breaks indeed Lorentz invariance but preserves CPT and gauge invariance. The total Lagrangian density is then

$$
\mathcal{L}=-\frac{1}{4} F^{\mu \nu} F_{\mu \nu}+\bar{\psi}\left[\gamma^{\mu}\left(i \partial_{\mu}-e A_{\mu}\right)-m\right] \psi-\frac{1}{4}\left(k_{F}\right)_{\mu \nu \rho \sigma} F^{\mu \nu} F^{\rho \sigma},
$$

where the first term gives the standard propagation of the photon, with Maxwell field strength $F_{\mu \nu} \equiv \partial_{\mu} A_{\nu}-\partial_{\nu} A_{\mu}$, the second term gives the standard propagation of a Dirac fermion (mass $m$ and electric charge $e$ ) and its standard minimal interaction with the photon field $A_{\mu}$, and the last term modifies the photon propagation by adding a dimension-four operator for CPT-even Lorentz violation [15, 16]. The Minkowski metric $g_{\mu \nu}(x)=\eta_{\mu \nu} \equiv$ $[\operatorname{diag}(+1,-1,-1,-1)]_{\mu \nu}$ and natural units with $\hbar=1=c$ are used throughout this article (see below for a remark on the meaning of the velocity $c$ ).

The constant fixed "tensor" $\left(k_{F}\right)_{\mu \nu \rho \sigma}$ has 20 independent components, ten of which produce birefringence and eight of which lead to direction-dependent modifications of the photon-propagation properties. The remaining two components correspond to an isotropic modification of the photon propagation and an unobservable double trace that changes the 
normalization of the photon field. The isotropic nonbirefringent violation of Lorentz invariance in the photon sector is then controlled by a single dimensionless parameter $\kappa$, which enters the fixed tensor $k_{F}$ of (2.1) in the following way:

$$
\left(k_{F}\right)^{\lambda}{ }_{\mu \lambda \nu}=\frac{\kappa}{2}[\operatorname{diag}(3,1,1,1)]_{\mu \nu} .
$$

Notice that the Ansatz (2.2) gives $\left(k_{F}\right)^{\mu \nu}{ }_{\mu \nu}=0$, which removes the unobservable double trace. In physical terms, the velocity $c$ corresponds to the maximum attainable velocity of the massive Dirac fermion of (2.1), whereas the phase velocity of the photon is given by

$$
v_{\mathrm{ph}}=\frac{\omega}{|\vec{k}|}=\sqrt{\frac{1-\kappa}{1+\kappa}} c,
$$

which is smaller/larger than $c$ for positive/negative values of $\kappa$.

As mentioned in the Introduction, isotropic Lorentz-violating effects can also appear in the fermion sector. But, for a single fermion, isotropic $c$-type Lorentz-violating effects can be moved to the photon sector by a suitable change of spacetime coordinates [8] (see also App. B of Ref. [3]).

For nonzero values of the LV parameter $\kappa$ in the theory (2.1)-(2.2), certain processes conventionally forbidden become allowed. For positive $\kappa$, a Cherenkov-type radiative process can already occur in the vacuum: $f^{ \pm} \rightarrow f^{ \pm}+\widetilde{\gamma}$, with $f^{ \pm}$standing for an electrically-charged Dirac (anti-)fermion and $\widetilde{\gamma}$ denoting the nonstandard photon of the theory considered. Since such vacuum-Cherenkov processes remain unobserved so far, constraints on the positive value of this parameter have been determined, for example, by considering the physics of particle accelerators [17, 18]. Even better, cosmic-ray data have reduced the upper limit to [3]

$$
\kappa<6 \times 10^{-20} \quad(98 \% \text { C.L. }) .
$$

For negative $\kappa$ in the theory (2.1) $-(2.2)$, photons become unstable above the energy threshold

$$
\omega_{\mathrm{th}}=2 m \sqrt{\frac{1-\kappa}{-2 \kappa}}
$$

and decay into pairs of electrically charged fermions. Hereafter, we consider the Dirac fermion field $\psi$ to be the electron/positron field with the corresponding constants

$$
(m, e)=\left(m_{\text {electron }}, e_{\text {electron }}\right)
$$

The tree-level photon decay $(\mathrm{PhD})$ rate as a function of the photon energy $\omega \geq \omega_{\mathrm{th}}$ is given by $[3,4]$

$$
\Gamma_{\mathrm{PhD}}(\omega)=\frac{\alpha}{3} \frac{-\kappa}{1-\kappa^{2}} \sqrt{\omega^{2}-\omega_{\mathrm{th}}^{2}}\left(2+\omega_{\mathrm{th}}^{2} / \omega^{2}\right),
$$

where $\alpha \equiv e^{2} /(4 \pi) \approx 1 / 137$ is the fine-structure constant and $m \approx 511 \mathrm{keV}$ is the electron mass entering the threshold energy $\omega_{\text {th }}$ from (2.5) . 
As photons above threshold decay very efficiently into pairs $\left(\widetilde{\gamma} \rightarrow e^{-}+e^{+}\right)$, only belowthreshold photons will reach Earth from distant sources and will travel through the Earth atmosphere. For this reason, the observation of energetic gamma rays in the Earth atmosphere can be used to determine a bound on negative $\kappa$. In fact, the most stringent lower limit has been obtained from the terrestrial observation of gamma rays [3]

$$
-9 \times 10^{-16}<\kappa \quad(98 \% \text { C.L. })
$$

For completeness, we mention that an astrophysics lower bound of order $-6 \times 10^{-20}$ has been obtained by considering synchrotron radiation in the Crab Nebula [19] (the $\kappa$ bound quoted is from Ref. [4]). This astrophysics bound is, however, only qualitative, different from bound (2.8) with a specified confidence level. Moreover, bound (2.8) does not rely on assumptions about the astronomical source, the only input for the energy determination of the gamma ray being well-known physical processes operating in the environment of the Earth atmosphere.

Since the lower limit (2.8) from terrestrial observations of gamma-ray primaries is about four orders of magnitude weaker than the upper limit (2.4) from cosmic-ray primaries, we would like to find a process that could allow further improvements and that would be accessible to terrestrial observations (e.g., in the Earth atmosphere).

\section{EXTENSIVE AIR SHOWERS}

\section{A. Preliminary remarks}

We now present a new method to explore the negative range of values of the LV parameter $\kappa$ as defined in Sec. II] Even though gamma rays have already served as tools to study this part of parameter space, we present a novel method, in which above-threshold photons can be produced in particle cascades in the Earth atmosphere. We will show that Lorentz-violating effects for sufficiently large (but numerically still very small) values of $\kappa$ can drastically modify the development of the electromagnetic components of the shower.

When a cosmic-ray hadronic primary impacts the upper Earth atmosphere, charged and neutral pions are produced. The high-energy charged mesons usually continue to feed the hadronic shower, while the neutral pions rapidly decay into photons triggering an electromagnetic shower. The particle cascades produced by the interaction of the primary cosmic ray with the atmosphere are known as extensive air showers (EAS).

Since we are ultimately interested in the effects of modified electrodynamics, we focus on the development of the electromagnetic shower. Consider, first, the standard Lorentzinvariant theory (LV parameter $\kappa=0$ ). After two photons of energy $\omega_{0}$ are produced from the decay of neutral pions $\left(\pi^{0}\right)$, their propagation in the Coulomb fields of the atomic nuclei of the atmosphere will lead to the production of electron-positron pairs, the "external conversion" process. Subsequently, each charged lepton will produce new photons by the Bremsstrahlung process. These are the two relevant high-energy processes for the standard theory and they give an exponential multiplication of the number of particles in the electromagnetic shower. 


\section{B. Heitler model}

A detailed description of the shower development requires a numerical simulation of the particle production as well as a probabilistic distribution of energy between the daughter particles at each interaction point. Still, a general analytic description of the main properties of the electromagnetic shower can be obtained by use of the Heitler model (see Sec. 24 of Ref. [14] and references therein), a simplified representation of the processes that produce new photons and charged leptons in the shower. This simple model accurately describes the most important features of the electromagnetic component of the shower.

The Heitler model depicts the particle multiplication as a binary tree (cf. Fig. 27 of Ref. [14] and Fig. 2 of Ref. [20]):

- each photon produces two charged leptons via pair production in the presence of a nucleus,

- each new charged lepton produces a lepton and a photon via Bremsstrahlung.

Consider an initial photon of energy $\omega_{0}$. After $n$ generations, the shower will then contain $2^{n}$ particles and the energy of the parent particle will be split in equal parts, each particle having an energy

$$
E_{n}=\omega_{0} / 2^{n}
$$

The particle multiplication will continue until the individual particle energy reaches a critical value $E_{c}$ and energy loss by ionization starts to dominate over radiative processes. In air, the critical value $E_{c}$ is of order [20]

$$
E_{c} \approx 80 \mathrm{MeV}
$$

Using the similarity of the pair-production and Bremsstrahlung cross sections, the interaction step length $d$ of charged leptons and photons can be approximated as being equal. Hence, the shower reaches its maximum after propagating in the Earth atmosphere to a depth

$$
X_{\max }=\lambda_{r} \ln \left(\omega_{0} / E_{c}\right),
$$

where $\lambda_{r} \equiv d / \ln 2$ is the radiation length in the medium. For air, we have [20]

$$
\lambda_{r} \approx 37 \mathrm{~g} / \mathrm{cm}^{2} \text {. }
$$

The depth of the shower maximum (3.3) is a quantity that cosmic-ray observatories such as the Pierre Auger Observatory [21] can measure reliably. For $10^{19} \mathrm{eV}$ showers observed by Auger, the systematic uncertainty in $X_{\max }$ is approximately $10 \mathrm{~g} / \mathrm{cm}^{2}$ and the resolution is approximately $15 \mathrm{~g} / \mathrm{cm}^{2}$ [22].

\section{Shower model with Lorentz violation}

Consider, next, the effects from the Lorentz-violating modification in (2.1) on the development of the electromagnetic shower. In the presence of isotropic nonbirefringent Lorentzviolating electrodynamics (2.2), the development of the shower will be modified by nonstandard decay processes which become allowed. For a negative value of the LV parameter $\kappa$, 
in particular, photons above the threshold energy (2.5) become unstable and rapidly decay into electron-positron pairs. The expression for the decay rate has already been given in (2.7).

First, take the LV parameter $\kappa$ to have the numerical value $-9 \times 10^{-16}$, which is consistent with the quantitative bound (2.8). Figure1 shows the photon decay length $l_{\mathrm{PhD}} \equiv 1 / \Gamma_{\mathrm{PhD}}$ in meters for this value of $\kappa$ (the result for a $10^{4}$ times smaller value of $\kappa$ is also shown and will be discussed later). The figure makes clear that photon decay is a very efficient energy-loss mechanism: above-threshold photons from astrophysical sources cannot reach Earth as they promptly decay into charged leptons. The corresponding threshold energy for photon decay into an electron-positron pair is $\omega_{\text {th }} \approx 24 \mathrm{TeV}$, as follows from (2.5) with $\kappa=-9 \times 10^{-16}$ and $m=m_{\text {electron }} \approx 511 \mathrm{keV}$.

Very energetic cosmic-ray hadronic primaries can have energies several orders of magnitude above this $24 \mathrm{TeV}$ threshold. If about $10 \%$ of the primary energy gets passed along to the first photons in the EAS through neutral-pion decay (see App. A for further remarks on this decay process in the LV theory), these photons initiating the electromagnetic cascade will be above threshold. The standard characteristic radiation distance for pair production in air is of the order of kilometers, whereas Fig. 1 shows that a photon with an energy above threshold will decay after a few millimeters or less (or after some $10 \mathrm{~cm}$ or less for a $10^{4}$ times smaller value of $\kappa$ ). The subsequent electromagnetic shower would be drastically modified because these photons would promptly produce electron-positron pairs via photon decay rather than the conventional pair-production mechanism. In the following generations, a charged lepton produces a Bremsstrahlung photon with energy $\omega>\omega_{\text {th }}$, which will almost immediately decay into a new pair of charged leptons. We can call these "PhD-leptons," in order to distinguish them from the charged leptons (called "Br-leptons") which are produced with the Bremsstrahlung emission of photons.

Inspired by the Heitler model described in Sec. IIIB, we follow a similar approach to present a general description of the main features of the modified shower. In our case, the shower proceeds as follows, with $\widetilde{\gamma}$ denoting the nonstandard photon described by the theory (2.1) $-(2.2)$ with negative LV parameter $\kappa$.

A $10^{15} \mathrm{eV}-10^{20} \mathrm{eV}$ cosmic-ray hadronic primary impinges on the Earth atmosphere, producing charged and neutral pions. Photons from subsequent neutral-pion decays $\left(\pi^{0} \rightarrow\right.$ $2 \widetilde{\gamma})$ will then have energies above the threshold (2.5) and will promptly decay into electronpositron pairs $\widetilde{\gamma} \rightarrow e^{-}+e^{+}$. Each of these PhD-leptons $\left(e^{ \pm}\right)$will produce a Br-lepton and a photon, with the latter rapidly decaying into a new PhD-lepton pair. The combined process is then given by

$$
e^{ \pm} \rightarrow e^{ \pm}+\widetilde{\gamma} \Rightarrow e^{ \pm}+\left(e^{-}+e^{+}\right)
$$

where the double arrow indicates the prompt nonstandard decay of $\widetilde{\gamma}$. Note that, for the small values of $\kappa$ considered, the rate of the first (Bremsstrahlung) process in (3.5) is essentially the same as in the Lorentz-invariant theory.

Since the photon decay in (3.5) occurs almost immediately after the Bremsstrahlung emission, effectively three charged leptons are produced at each generation, which share the energy of the parent particle. Let us assume that, at each generation, three leptons are produced: one Br-lepton and two PhD-leptons, each having one-third of the total energy. 
Notice that this is an approximate scenario because, even in an equal-energy-share situation, the Br-lepton would take half of the parent's energy, while the two PhD-leptons would each take one-fourth of the parent's energy. Still, this very simplified model allows an analytic description of the shower that provides a general insight into the way the modified shower will develop.

A photon with energy $\omega_{0} \gg \omega_{\text {th }}$ rapidly decays into two PhD-leptons, each having an equal energy $\omega_{0} / 2$ by assumption. Then, each of these two leptons develops a branch whose number of particles grows as $3^{n^{\prime}-1}$, each with energy

$$
E_{n^{\prime}}=\frac{\omega_{0} / 2}{3^{n^{\prime}-1}}
$$

Since the first photon decay occurs immediately, the parent photon with energy $\omega_{0} \gg \omega_{\text {th }}$ barely propagates through the atmosphere before decaying. Hence, the depth of the shower in the atmosphere is given by $X=\left(n^{\prime}-1\right) d$. The particle multiplication in the shower continues in this manner until the particles reach an energy below the threshold $\omega_{\text {th }}$ for photon decay. After this, the shower continues in the conventional way as described in Sec. IIIB. For $\omega_{0} / 2>\omega_{\text {th }}>E_{c}$, the shower-maximum depth of this modified shower in the atmosphere can then be written as the following sum:

$$
\widetilde{X}_{\max }=\lambda_{r} \eta \ln \left(\frac{\omega_{0} / 2}{\omega_{\mathrm{th}}}\right)+\lambda_{r} \ln \left(\frac{\omega_{\mathrm{th}}}{E_{c}}\right),
$$

with

$$
\eta \equiv \ln 2 / \ln 3
$$

For $\omega_{0}<\omega_{\text {th }}$, the shower-maximum depth is given by the standard result (3.3). For intermediate values, $\omega_{\text {th }} \leq \omega_{0} \leq 2 \omega_{\text {th }}$, the function $X_{\max }\left(\omega_{0}\right)$ interpolates between the standard behavior (3.3) and the nonstandard behavior (3.7).

The first term in (3.7) describes the part of the shower above the threshold energy (2.5) and the second term in (3.7) describes the part of the shower which begins with this threshold energy and ends when the energy per particle falls below $E_{c}$ given by (3.2). The factor $\eta$ in the first term of (3.7) traces back to the fact that each generation of the LV shower model produces 3 particles, whereas each generation of the standard Lorentz-invariant shower model of Sec. IIIB produces only 2 particles.

The calculated $\widetilde{X}_{\max }$ from (3.7) is shown in Fig. 2 for two values of $\kappa$ in comparison to the standard $X_{\max }$ from (3.3). We see a significant reduction of the depth of shower maximum. For instance, for $\omega_{0}=10^{9} \mathrm{GeV}$, the depth of shower maximum is reduced by some $160 \mathrm{~g} / \mathrm{cm}^{2}$ for $\kappa=-9 \times 10^{-16}$ and some $100 \mathrm{~g} / \mathrm{cm}^{2}$ for $\kappa=-9 \times 10^{-20}$. Such reductions are large compared to the experimental uncertainties. In addition, it is interesting to note that the factor $\eta$ in (3.7) diminishes the slope (or "elongation rate") of the shower-maximum depth as a function of $\ln \omega_{0}$.

The expression (3.7) for the modified shower-maximum depth can also be written as a change of the conventional depth (3.3),

$$
\widetilde{X}_{\max }=(1+\delta) X_{\max },
$$


with

$$
\delta=\frac{(\eta-1) \ln \left(\omega_{0} / \omega_{\mathrm{th}}\right)-\eta \ln 2}{\ln \left(\omega_{0} / E_{c}\right)} .
$$

Since $\omega_{0} \gg \omega_{\text {th }}$ by assumption and $\eta<1$ by definition, we find that $\delta$ is negative, which implies that the shower-maximum depth gets reduced. This feature is to be expected because the particle multiplication (and corresponding energy share) occurs faster in the Lorentzviolating case than in the conventional case.

Figure 3 shows this relative modification of the shower-maximum depth for different values of the LV parameter $\kappa$. Expression (3.10) relates the fractional depth change of the electromagnetic shower to the energy of the initiating photon $\omega_{0}$. This expression can be inverted for $\kappa$ as a function of the photon energy $\omega_{0}$, for a given precision $\delta$ in the measurement of the shower-maximum depth. Figure 4 shows the potential sensitivity to the LV parameter $\kappa$ obtained from different values of the relative modification $\delta$ of the shower-maximum depth.

Figures 3 and 4 show that the shower-maximum depth is particularly sensitive to a negative value of the Lorentz-violating parameter $\kappa$. A reliable estimate of the energy $\omega_{0}$ of the first photon and a careful determination of the allowed deviation from the standard $X_{\max }$ behavior could improve the existing $\kappa$ limit on the negative side by several orders of magnitude. For example, the absence of deviations from the conventional maximum depth within $5 \%$ for initial photon energies $\omega_{0}$ of order $10^{7} \mathrm{GeV}$ would increase the excluded parameter space by four orders of magnitude. In that case, the allowed range of negative values of the LV parameter $\kappa$ would be reduced significantly and would become similar to the allowed range (2.4) of positive values obtained from the observation of energetic cosmic rays.

\section{SUMMARY}

In this article, we considered a theory with isotropic nonbirefringent Lorentz violation in the photon sector and studied the effects on the development of extensive air showers. In particular, we focused on the consequences of a negative value for the Lorentz-violating parameter $\kappa$ because positive values are already well constrained [3].

The relevant nonstandard process for $\kappa<0$ is photon decay into an electron-positron pair [3], which substantially modifies the development of the electromagnetic component of the shower. Since the photon decay length is of the order of a meter or less, photons produced by the decay of neutral pions or by subsequent Bremsstrahlung emission will promptly decay into electron-positron pairs, well before conventional pair production can take place. This nonstandard decay process accelerates the particle multiplication in the shower, which reaches its maximum earlier than for the conventional Lorentz-invariant case.

Two stages are identified in this electromagnetic shower. In the first stage, the rapid Lorentz-violating decay of Bremsstrahlung-photons produced with energies above the photon-decay threshold will effectively lead to the production of three charged leptons at each generation, one "Bremsstrahlung-lepton" and two "photon-decay-leptons." This part of the shower contains mostly electrons and positrons, and the energy per particle 
will rapidly fall below the threshold for photon decay. In the second stage, the particle multiplication proceeds in the conventional manner. The energy per particle starts at the Lorentz-violating photon-decay threshold energy and drops until it is below the critical value $E_{c}$, at which moment the particle number in the shower reaches its maximum.

We have found that negative $\kappa$ could drastically reduce the shower-maximum depth $X_{\max }$ of the electromagnetic shower as well as modify its energy dependence. The absence of these features could be used to significantly improve the existing limits on negative values of $\kappa$.

\section{ACKNOWLEDGMENTS}

We thank B. Altschul and D. Kostunin for useful comments on an earlier version of this article. This work was supported in part by the German Research Foundation (DFG) under Grants No. KL 1103/4-1 and No. RI 1838/6-1.

\section{Appendix A: Heuristics of Lorentz-violating decays}

In this appendix, we briefly discuss the heuristics of certain decay processes in the isotropic modified Maxwell theory (2.1)-(2.2) based on the concept of the effective mass-square [2325]. In particular, we make, as promised in Sec. IIIC, some remarks on neutral-pion decay in the Lorentz-violating (LV) theory considered, $\pi^{0} \rightarrow \widetilde{\gamma} \widetilde{\gamma}$

From the dispersion relation implicit in (2.3) with $c=1$, we have the following definition of the effective mass-square:

$$
\omega^{2}=\frac{1-\kappa}{1+\kappa}|\vec{k}|^{2} \sim|\vec{k}|^{2}+(-2 \kappa)|\vec{k}|^{2} \equiv|\vec{k}|^{2}+m_{\mathrm{eff}, \widetilde{\gamma}}^{2}(\vec{k})
$$

In four-vector notation, this reads

$$
k^{2}=\omega^{2}-|\vec{k}|^{2} \sim m_{\mathrm{eff}, \widetilde{\gamma}}^{2}(\vec{k}) \equiv-2 \kappa|\vec{k}|^{2} .
$$

The 3-momentum dependence of the effective mass-square in (A2) makes clear that the theory is Lorentz violating (in the following, this 3-momentum dependence is often kept implicit). As discussed extensively in Ref. [25], the effective mass-square allows for a heuristic discussion of LV decay processes in general. Here, we consider three decay processes in the isotropic modified Maxwell theory with negative $\kappa$.

The first process is photon decay into an electron-positron pair [3], $\widetilde{\gamma} \rightarrow e^{-}+e^{+}$. The threshold photon energy from (2.5) becomes for small $|\kappa|$

$$
\omega_{\mathrm{th}} \sim 2 m \sqrt{\frac{1}{-2 \kappa}},
$$

which reads as follows in terms of the effective mass from (A2):

$$
m_{\mathrm{eff}, \widetilde{\gamma}}^{\text {(th) }} \sim 2 m .
$$

Hence, the LV photon decay is as if a massive scalar at rest decays into two scalars of mass $m$. 
The second process is Bremsstrahlung by an electron or positron in the Coulomb field of a nucleus, $e^{ \pm}(+Z) \rightarrow e^{ \pm}+\widetilde{\gamma}(+Z)$. Below (3.5) in the main text, we have already mentioned that the rate would be essentially the same as in the Lorentz-invariant theory. Indeed, consider energy conservation (3-momentum conservation is taken care of by the nucleus),

$$
\sqrt{E^{2}+M_{e}^{2}}=\sqrt{E^{\prime 2}+M_{e}^{2}}+\sqrt{|\vec{k}|^{2}+m_{\mathrm{eff}, \widetilde{\gamma}}^{2}}
$$

with initial electron energy $E$, final electron energy $E^{\prime}$, and photon 3 -momentum $\vec{k}$. Considering ultrarelativistic initial and final electrons and using the effective mass-square from (A2), we get

$$
E \sim E^{\prime}+|\vec{k}|(1-\kappa)
$$

A typical process at ultrahigh energies can have $E^{\prime}=E / 2$ with the photon taking the remaining energy $E / 2$, just as for the case of the standard Lorentz-invariant theory (see Fig. 14 on p. 170 in Ref. [6] ).

The third process is neutral-pion decay in the theory with the Standard Model action augmented by a single LV photonic term proportional to $\kappa$ (taken to be negative and to have a very small magnitude). The energies and 3-momenta of the process are denoted as follows:

$$
\pi^{0}(E, \vec{q}) \rightarrow \widetilde{\gamma}(\omega, \vec{k})+\widetilde{\gamma}\left(\omega^{\prime}, \vec{k}^{\prime}\right)
$$

with the on-shell energies $E(\vec{q})=\sqrt{|\vec{q}|^{2}+\left(m_{\pi^{0}}\right)^{2}}$ and $\omega(\vec{k}) \geq 0$ from (A1). In terms of the photon effective mass-square from (A2), we have the cutoff condition

$$
m_{\pi^{0}} \sim 2 m_{\text {eff, } \widetilde{\gamma}}^{\text {(cutoff })} \sim \sqrt{-2 \kappa}\left(2 \omega_{\text {cutoff }}\right)
$$

This gives, upon identifying the pion energy $E \sim 2 \omega$, the following cutoff energy for neutralpion decay:

$$
E_{\pi^{0}}^{(\text {cutoff })} \sim \frac{m_{\pi^{0}}}{\sqrt{-2 \kappa}}
$$

which agrees with the kinematic result of Ref. 24]. The heuristics of the first part of (A8) makes clear that neutral-pion decay into two LV photons $\widetilde{\gamma}$ can occur for pion energies below (A9) but not above.

The general expression for the neutral-pion decay parameter $\gamma[25]$ is as follows (see, e.g., Sec. 3.6 of Ref. [26]):

$$
\gamma_{\pi^{0} \rightarrow \widetilde{\gamma}+\widetilde{\gamma}}(\vec{q})=\frac{1}{2} \frac{1}{(2 \pi)^{2}} \int \frac{d^{3} k}{2 \omega(\vec{k})} \int \frac{d^{3} k^{\prime}}{2 \omega\left(\vec{k}^{\prime}\right)} \delta^{4}\left(q-k-k^{\prime}\right)|\mathcal{M}|^{2},
$$

with the symmetry factor $1 / 2$ and the matrix element $\mathcal{M}$. Observe that, in a Lorentzinvariant theory, the right-hand side of (A10) is manifestly Lorentz invariant and $\gamma$ is the 
decay constant, which is independent of $\vec{q}$. Taking the effective pion-photon-photon interaction (from anomalous triangle diagrams with quarks) to be [26],

$$
\mathcal{L}_{\text {eff }}=\alpha C \phi \epsilon^{\alpha \beta \gamma \delta} F_{\alpha \beta} F_{\gamma \delta}
$$

with coupling constant $C$ of mass dimension -1 , the phase-space integral of the LV decay parameter (A10) gives

$$
\gamma_{\pi^{0} \rightarrow \tilde{\gamma}+\widetilde{\gamma}}(\vec{q})= \begin{cases}\frac{1}{2} \frac{1}{8 \pi}\left(\frac{1-\kappa}{1+\kappa}\right)^{3 / 2}\left[(8 \alpha C)^{2} \frac{1}{2}\left(m_{\pi^{0}}\right)^{4}+\cdots\right] & \text { for } E(\vec{q})<E_{\pi^{0}}^{\text {(cutoff) }}, \\ 0 & \text { otherwise, }\end{cases}
$$

with the cutoff energy from (A9). The ellipsis in the large square bracket of (A12) is because the result for $|\mathcal{M}|^{2}$ depends on the LV parameter $\kappa$, as the calculation involves, for example, the $k^{2}$ of the photons (see, e.g., Secs. 4.5 and 7.1 of Ref. [26] for an extensive discussion of the standard Lorentz-invariant decay process). The complete result for (A12) will be given elsewhere [27].

Regarding the neutral-pion-decay process, let us briefly turn to extensive-air-shower phenomenology in our LV theory. The neutral-pion-decay cutoff energy (A9) is about a factor 100 larger than the photon-decay threshold energy (2.5), because of the different masses involved. For a given negative value of $\kappa$, there will be no photons produced from neutralpion decay with energies $\omega_{0}>1 / 2 \times E_{\pi^{0}}^{\text {(cutoff) }}$. If we now look at Fig. 2 and translate the initial photon energy $\omega_{0}$ on the horizontal axis into a typical energy of a hadronic primary (very roughly, $E_{\text {prim }} \sim 10 \times \omega_{0}$ ), then the $\kappa \neq 0$ curves for $X_{\max }$ will, most likely, receive an additional suppression for $E_{\text {prim }} \gtrsim 10 \times E_{\pi^{0}}^{\text {(cutof) }}$. These and other effects must be taken care of in a numerical simulation, on which we plan to report in a forthcoming publication.

[1] F.R. Klinkhamer and M. Risse, "Ultra-high-energy cosmic-ray bounds on nonbirefringent modified-Maxwell theory,” Phys. Rev. D 77, 016002 (2008), arXiv:0709.2502.

[2] F.R. Klinkhamer and M. Risse, "Addendum: Ultrahigh-energy cosmic-ray bounds on nonbirefringent modified-Maxwell theory," Phys. Rev. D 77, 117901 (2008), arXiv:0806.4351.

[3] F.R. Klinkhamer and M. Schreck, "New two-sided bound on the isotropic Lorentz-violating parameter of modified Maxwell theory," Phys. Rev. D 78, 085026 (2008), arXiv:0809.3217.

[4] J.S. Díaz and F.R. Klinkhamer, "Parton-model calculation of a nonstandard decay process in isotropic modified Maxwell theory," Phys. Rev. D 92, 025007 (2015), arXiv:1504.01324.

[5] V.A. Kostelecký, and M. Mewes, "Lorentz-violating electrodynamics and the cosmic microwave background," Phys. Rev. Lett. 99, 011601 (2007), astro-ph/0702379.

[6] J.S. Díaz, V.A. Kostelecký, and M. Mewes, "Testing relativity with high-energy astrophysical neutrinos," Phys. Rev. D 89, 043005 (2014), arXiv:1308.6344.

[7] J.S. Díaz and T. Schwetz, "Limits on CPT violation from solar neutrinos," Phys. Rev. D 93, 093004 (2016), arXiv:1603.04468.

[8] Q. Bailey and V.A. Kostelecký, "Lorentz-violating electrostatics and magnetostatics," Phys. Rev. D 70, 076006 (2004), arXiv:hep-ph/0407252. 
[9] J.P. Noordmans, C.J.G. Onderwater, H.W. Wilschut, and R.G.E. Timmermans, "Question of Lorentz violation in muon decay," Phys. Rev. D 93, 116001 (2016), arXiv:1412.3257.

[10] E.E. Antonov et al., "Test of Lorentz invariance through observation of the longitudinal development of ultrahigh-energy extensive air showers," JETP Lett. 73, 446 (2001).

[11] B. Altschul, "Astrophysical limits on Lorentz violation for pions," Phys. Rev. D 77, 105018 (2008), arXiv:0712.1579.

[12] D. Boncioli et al., "Future prospects of testing Lorentz invariance with UHECRs," in: Proceedings of the 34th International Cosmic Ray Conference, PoS ICRC2015 (2015), arXiv:1509.01046.

[13] J.S. Díaz, "Neutrinos as probes of Lorentz invariance," Adv. High Energy Phys. 2014, 962410 (2014), arXiv:1406.6838.

[14] W. Heitler, Quantum Theory of Radiation, second edition (Oxford University Press, London, 1944).

[15] S. Chadha and H.B. Nielsen, "Lorentz invariance as a low-energy phenomenon," Nucl. Phys. B 217, 125 (1983).

[16] V.A. Kostelecký and M. Mewes, "Signals for Lorentz violation in electrodynamics," Phys. Rev. D 66, 056005 (2002), arXiv:hep-ph/0205211.

[17] M.A. Hohensee, R. Lehnert, D.F. Phillips, and R.L. Walsworth, "Particle-accelerator constraints on isotropic modifications of the speed of light," Phys. Rev. Lett. 102, 170402 (2009), arXiv:0904.2031.

[18] B. Altschul, "Bounding isotropic Lorentz violation using synchrotron losses at LEP," Phys. Rev. D 80, 091901 (2009), arXiv:0905.4346.

[19] B. Altschul, "Lorentz violation and synchrotron radiation," Phys. Rev. D 72, 085003 (2005), arXiv:hep-th/0507258.

[20] A. Letessier-Selvon and T. Stanev, "Ultrahigh energy cosmic rays," Rev. Mod. Phys. 83, 907 (2011), arXiv:1103.0031.

[21] A. Aab et al. [Pierre Auger Collaboration], "The Pierre Auger Cosmic Ray Observatory," Nucl. Instrum. Meth. A 798, 172 (2015), arXiv:1502.01323.

[22] A. Aab et al. [Pierre Auger Collaboration], "Depth of maximum of air-shower profiles at the Pierre Auger Observatory. I. Measurements at energies above $10^{17.8} \mathrm{eV}, "$ Phys. Rev. D 90, 122005 (2014), arXiv:1409.4809.

[23] E. Strakhov and D.A. Owen, "Decay of a Chern-Simons 'photon' and $e^{+} e^{-} \mathrm{MeV}$ peaks," J. Phys. G 22, 473 (1996).

[24] S.R. Coleman and S.L. Glashow, "High-energy tests of Lorentz invariance," Phys. Rev. D 59, 116008 (1999), arxiv:hep-ph/9812418.

[25] C. Kaufhold and F. R. Klinkhamer, "Vacuum Cherenkov radiation and photon triple-splitting in a Lorentz-noninvariant extension of quantum electrodynamics," Nucl. Phys. B 734, 1 (2006), arXiv:hep-th/0508074.

[26] B. de Wit and J. Smith, Field Theory in Particle Physics, Volume 1 (North-Holland Publ., Amsterdam, 1986).

[27] F.R. Klinkhamer, "Lorentz-violating neutral-pion decays in isotropic modified Maxwell theory," arXiv:1610.03315. 


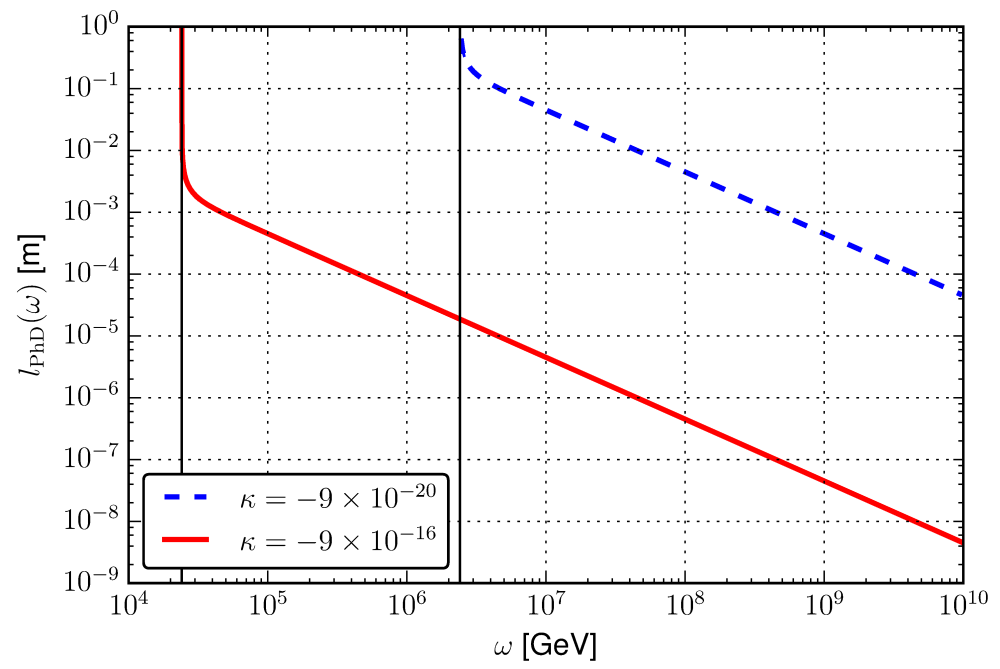

FIG. 1. Photon decay length $l_{\mathrm{PhD}} \equiv 1 / \Gamma_{\mathrm{PhD}}$ as a function of the photon energy $\omega$ for the process $\widetilde{\gamma} \rightarrow e^{-}+e^{+}$. Two values of the Lorentz-violating parameter $\kappa$ are considered, $\kappa=-9 \times 10^{-16}$ and $\kappa=-9 \times 10^{-20}$, and the vertical lines indicate the respective threshold energies, defined by (2.5) in terms of $\kappa$ and the electron mass $m$. For $\kappa \uparrow 0$, the photon-decay threshold energy moves towards infinity.

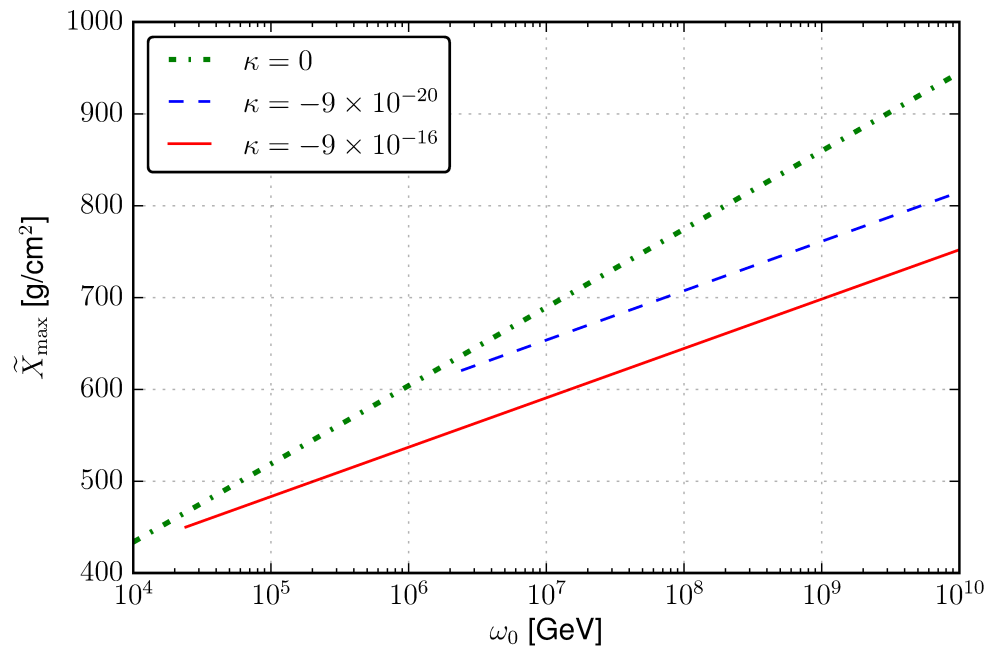

FIG. 2. Shower-maximum depth (3.7) of the electromagnetic shower for different values of $\kappa$ as a function of the initial photon energy $\omega_{0}$. The blue-dashed curve for $\kappa=-9 \times 10^{-20}$ has a larger photon-decay threshold energy than the red-solid curve for $\kappa=-9 \times 10^{-16}$. 


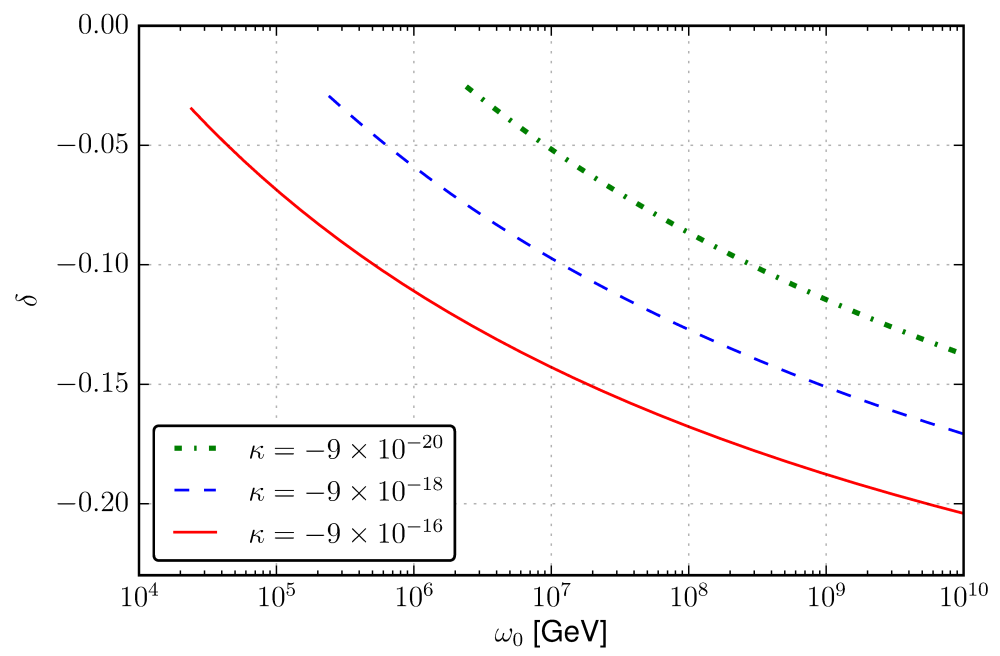

FIG. 3. Relative modification (3.10) of the shower-maximum depth (3.9) for different values of the Lorentz-violating parameter $\kappa$. If an electromagnetic shower is initiated by a $10^{7}-\mathrm{GeV}$ photon, a reduction of approximately $15 \%$ on $X_{\max }$ is expected from the current limit value $\kappa=-9 \times 10^{-16}$, whereas a $10 \%$ reduction is expected from $\kappa=-9 \times 10^{-18}$ and a reduction by $5 \%$ from $\kappa=$ $-9 \times 10^{-20}$. The three different curves start at different threshold energies defined by (2.5) in terms of $\kappa$ and the electron mass $m$.

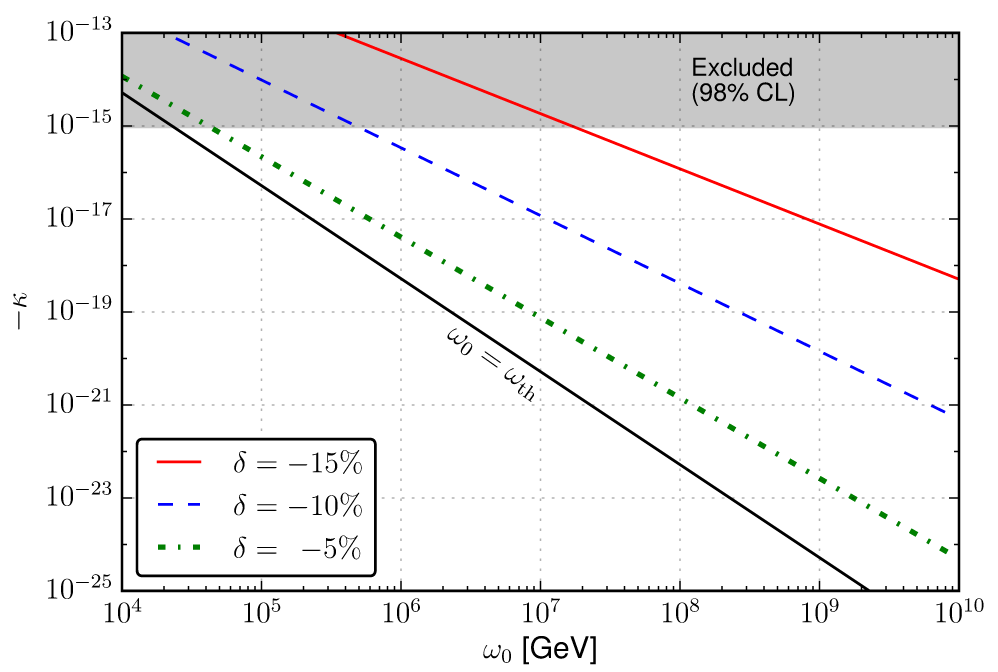

FIG. 4. Lorentz-violating parameter $\kappa$ as a function of the initial photon energy $\omega_{0}$ producing different relative modifications $\delta$ of the shower-maximum depth. Also shown is the line $\omega_{0}=$ $\omega_{\mathrm{th}}(\kappa)$ with $\omega_{\mathrm{th}}(\kappa)$ given by (2.5) and only photons to the right of this line are considered. If an electromagnetic shower is initiated by a $10^{8}-\mathrm{GeV}$ photon, a reduction of $15 \%$ on $X_{\max }$ would be produced by $\kappa \sim-10^{-16}$, a reduction of $10 \%$ by $\kappa \sim-5 \times 10^{-19}$, and a reduction of $5 \%$ by $\kappa \sim-2 \times 10^{-21}$. 